\title{
Breath-Actuated Nebulizer for Patients With Exacerbation of COPD: Efficiency and Cost
}

Aerosolized bronchodilators have been the first line therapy for exacerbation of COPD (ECOPD) to relieve bronchoconstriction and shortness of breath. Administration of bronchodilators in acute settings is commonly done with small-volume nebulizers, which deliver higher inhaled drug mass, compared to other devices such as pressurized metered-dose inhaler (pMDI) and dry powder inhaler (DPI). ${ }^{1}$ Small-volume nebulizers (SVNs), according to design, are categorized as constant-output, breath-enhanced, and dissymmetric (or breath-actuated). ${ }^{2}$ The breath-actuated nebulizer (BAN) produces aerosol during inspiration, when the negative pressure generated by the patient is sufficient to pull the actuator down into position, sealing the jet nozzle to allow medication to be drawn from the reservoir, generating aerosol. Studies report greater inhaled dose, less environmental contamination, and smaller particle size with the BAN, associated with 2-3 fold increased treatment time, than with other types of jet nebulizers. ${ }^{2,3}$ In a study in this issue of RespiRatory CARE, Haynes compared the BAN to continuous flow SVNs in patients with ECOPD. ${ }^{4}$ This represents the first study examining the clinical response of combined short acting $\beta$ adrenergic and anticholinergic bronchodilators from the BAN and the SVN in ECOPD by assessing inspiratory capacity (IC) and dyspnea via the Borg scale.

See the Original Study on Page 1385

This study employed a prospective randomized controlled trial design. Patients diagnosed with ECOPD were randomized to receive $2.5 \mathrm{mg}$ albuterol sulfate and $0.5 \mathrm{mg}$ ipratropium bromide in standard unit dose from either a BAN or a continuous SVN every 4 hours. Efforts were made to blind the data collection. Inspiratory capacity, dyspnea scale, and other physiological data, before and approximately 22 hours after enrollment were recorded and compared. The author reports that the BAN group had a greater change in IC and lower respiratory rate than the SVN group; however, there was no difference in resting dyspnea scale or stay.

\section{Physiological Response to Bronchodilator}

During ECOPD, small airways obstruction causes incomplete expiration and worsens hyperinflation, which can be measured by spirometry. As the functional residual volume increases, the IC decreases. ${ }^{5}$ With a reduction in IC, additional work is required to achieve sufficient tidal volume, which may induce the unpleasant sensation of dyspnea. ${ }^{6}$ There are correlations among hyperinflation, reduced IC, and worsened dyspnea sensation. Moreover, patients of ECOPD may have an increased respiratory rate.

The Haynes study shows that improved IC and lower respiratory rate may be the outcome of bronchodilator therapy delivered by the BAN, compared to standard continuous SVN. While the change in IC is significant, what does it signify? Are these finding of only academic interest, with no direct benefit to the patients? We would suggest that the implications of these findings extend beyond the limitation of the current study design. Now that the change in IC and respiratory rate has been observed, there may be value in determining the impact on exertional dyspnea (as opposed to the resting dyspnea scores studied).

Improvements of IC have been associated with reduced exertional dyspnea, which may be of benefit to this patient population, but further study would be required to substantiate this possibility. Exertional dyspnea has been associated with limitations in ability to exercise and perform activities of daily living. ${ }^{7}$ Consequently, if the improvement of IC over an extended period of time were associated with reduction in exertional dyspnea, it would be the first time that method of aerosol delivery was associated with an improvement in quality of life for COPD patients. Additional studies with an eye toward the ambulatory setting in patients with disabling COPD would be required to explore these possibilities.

\section{Efficacy Versus Cost}

In the hospital we tend to look at the price for the nebulizer and the medication, and the time of administration as cost, and weigh them against the "benefit" of completing an ordered therapy, with little differentiation among outcomes. There has been substantial interest in the use of the BAN, due to its increased inhaled efficiency of up to 3 -fold on drug delivery, but concerns have been raised about the longer treatment time required to administer standard dose volumes. In the acute care environment, where clinicians presumably stay at the bedside during adminis- 


\section{Breath-Actuated Nebulizer for Patients With Exacerbation of COPD}

tration, this increased time for administration has a negative economic impact, with increased labor costs. In an effort to reduce treatment times, clinicians and researchers have reported using higher concentration solutions, such as $2.5 \mathrm{mg}$ of albuterol in $1.0 \mathrm{~mL}$ with the BAN, rather than standard unit dose of albuterol (2.5 $\mathrm{mg}$ in $3.0 \mathrm{~mL})$. Several reports have compared using higher concentration formulations with the BAN to with standard dose volumes with the SVN, describing treatment times with the BAN that are less than the SVN, offsetting the higher price for the BAN. ${ }^{8}$ But what if the time of administration actually were to improve therapeutic benefit for the patient?

In contrast to previous clinical trials with the BAN, using concentrated bronchodilators in reduced dose volumes, this study represents the first apples-to-apples comparison between 2 devices, utilizing the same drug dose and dose volumes. The author advances several theories to explain the change in IC with the BAN, such as increased dose or differences in particle size, but studies with these inhaled bronchodilators suggest that differences in dose and small differences in particle size typically do not impact response in this population. An alternative hypothesis is that the greater time of administration with breath actuation may impact response. Although the time of treatment was not reported, it can be assumed that with the same dose volume the nebulization time was $2-3$-fold longer with the BAN than with the SVN. Could it be that the increased inhaled dose with prolonged treatment time combine to confer greater benefit?

If time of administration confers additional benefit, then we might need a different algorithm to assess cost/benefit for aerosol administration. If use of BAN with severe COPD were to increase IC and reduce exertional dyspnea better than other administration methods, then the benefits might substantially shift against the cost.

The total cost of a treatment with BAN may be increased, with the greater cost of the nebulizer and labor cost associated with longer treatment times. However, if there were a reduction in exertional dyspnea, the higher incremental costs might be less important than the impact on the ability of the patient to perform activities of daily

\footnotetext{
Dr Fink has disclosed relationships with Aerogen, Aridis, Cubist, Dance Pharma, Kalobios, and Novartis. The other authors have disclosed no conflicts of interest.
}

DOI: $10.4187 /$ respcare.02061 living. This could have greater implications in the home than in the hospital settings. In the ambulatory setting, where the time of administration is not an expense incurred by the patient, the question would become more of a question of patients tolerating a longer treatment time. Future studies should focus on impact of improved pulmonary function parameters on exertional dyspnea and the subsequent impact on activities of daily life and patient feelings of well being.

In conclusion, Haynes advances our understanding of using the BAN versus continuous SVN with an apples-toapples comparison of dose, identifying a difference in physiological effects in the ECOPD patient population. While not impacting clinical outcomes, the findings suggest a potential to impact patients in a way that has not previously been associated with difference in liquid aerosol devices, opening an array of questions for further studies.

Hui-Ling Lin MSc RRT RN Department of Respiratory Therapy College of Medicine Chang Gung University Taoyuan, Taiwan

James B Fink PhD RRT FAARC Division of Respiratory Therapy Georgia State University Atlanta, Georgia

\section{REFERENCES}

1. Rau JL. The inhalation of drugs: advantages and problems. Respir Care 2005;50(3):367-382.

2. Rau JL, Ari A, Restrepo RD. Performance comparison of nebulizer designs: constant-output, breath-enhanced, and dosimetric. Respir Care 2004;49(2):174-179.

3. Sangwan S, Condos R, Smaldone GC. Lung deposition and respirable mass during wet nebulization. J Aerosol Med 2003;16(4):379386.

4. Haynes JM. Randomized controlled trial of a breath-activated nebulizer in patients with exacerbation of COPD. Respir Care 2012; 57(9): 1385-1390

5. Ruppel GL. What is the clinical value of lung volumes? Respir Care 2012;57(1):26-35.

6. Puente-Maestu L, Stringer WW. Hyperinflation and its management in COPD. Int J Chron Obstruct Pulmon Dis 2006;1(4):381-400.

7. Mahler DA. Mechanisms and measurement of dyspnea in chronic obstructive pulmonary disease. Proc Am Thorac Soc 2006;3(3):234238 .

8. Arunthari V, Bruinsma RS, Lee AS, Johnson MM. A prospective, comparative trial of standard and breath-actuated nebulizer: efficacy, safety, and satisfaction. Respir Care 2012;57(8):1242-1247. 